\title{
Foreign Body in the Esophagus-Comparison Between Adult and Pediatric Population
}

\author{
Koirala K, ${ }^{1^{*}}$ Rai $S,{ }^{1}$ Chhetri $S,{ }^{1}$ Shah $R^{1}$ \\ ${ }^{1}$ Department of ENT, Manipal Teaching Hospital, Pokhara, Nepal
}

\section{*Corresponding Author: \\ Dr. Krishna Koirala MBBS, MS (ENT) \\ Department of ENT \\ MCOMS, Pokhara, Nepal \\ Email: gaukrishna@yahoo.com}

\section{Citation}

Koirala K, Rai S, Chettri S, Shah R. Foreign body in the esophagus- comparison between Adult and Paediatric population. Nepal Journal of Medical Sciences. 2012; 1(1): $42-44$.

\begin{abstract}
Background: Foreign body (FB) impaction in the esophagus is a common emergency in all age groups. In the children coins are the commonest foreign bodies while bones have been found as the commonest in adults.

Methods: This is a retrospective study comparing FB in the esophagus in adult and pediatric population between June 2007 and May 2010 in Manipal Teaching Hospital, Pokhara, Nepal. Most of the patients underwent rigid esophagoscopy and FB removal; few were removed under anesthesia by the anesthesiologists. Comparison was done in terms of pattern of $\mathrm{FB}$, their site of impaction and relative ease of removal. Data were collected from the case file available from the medical record department and analysed using SPSS 11.6.

Results: A total of 63 cases were included in this study. Out of which 28 (44.4\%) patients were children and 35 (55.5\%) were adults. Coin was the most common foreign body in the pediatric population (82.1\%) whereas bones were the commonest ones (91.4\%) in adults. In the pediatric population the commonest site of impaction was upper esophagus (92.8\%) whereas mid esophagus was the commonest site $(65.7 \%)$ in adults.
\end{abstract}

Conclusion:There are differences in various aspects of impacted foreign bodies in esophagus in adult and pediatric populations. Coins being the commonest foreign bodies in children are relatively easier to remove due to their higher location and less chances of trauma. Contrary in the adults, bones being the commonest foreign body are difficult to remove due to the lower location and chances of trauma.

Keywords: Foreign body; esophagus; rigid bronchoscopy

\section{Background:}

F oreign body stuck in the esophagus is a common entity in emergency practice. Small children have a common curiosity of putting objects into their mouth when they find them. It is even difficult for them to control the foreign body because of lack of posterior dentition. Foreign body in adults is mainly accidental. People with derranged mental status might ingest a foreign body that might get stuck for a prolonged period as a neglected one. Although common in children, they are not uncommon in adults. Recurrent episodes of foreign body impaction are common due to structural anomaly in the esophageal lumen or compression from outside the lumen. Coins are the commonest FB in children while bone are more common in adult population. ${ }^{1-5}$ This study was carried out to compare the incidence and varieties of foreign bodies stuck in the esophagus in pediatric and adult population that presented in our hospital. 


\section{Methods:}

This is a retrospective study carried out in the department of Otolaryngology and Head \& Neck Surgery, Manipal College of Medical Sciences, Pokhara, Nepal between June 2007 and May 2010. All cases were thoroughly evaluated including history, head and neck examination and imaging immediately before surgery. The imaging technique was $\mathrm{x}-$ ray soft tissue neck AP and lateral view for all the patients. The operative procedure was rigid esophagoscopy and foreign body removal under general anesthesia for all the patients. Data were taken from case files and analyzed using SPSS 11.6.

\section{Results:}

A total of 63 cases were included in this study. Out of which $28(44.4 \%)$ patients were less than 15 years and $35(55.5 \%)$ patients were above 15 years of age. Mean age of the patients in pediatrics and adult groups were 7.7 years $(\mathrm{SD}=4.23)$ and $43.05(\mathrm{SD}=20.64)$ years respectively. The age of the patients was ranging from 6 months to 81 years. There were 24 males and 39 females with male to female ratio of 0.62:1. In the pediatric population 17 patients were female and 11 were male $(\mathrm{M}: \mathrm{F}=0.65: 1)$ while in the adult population 22 were females and 13 were males $(\mathrm{M}: \mathrm{F}=0.59: 1)$. Rigid esophagoscopy was performed in 60 patients and it was $100 \%$ successful to remove the foreign bodies. In three of the pediatric populations, foreign bodies were removed by the anesthesiologists with Magill's forceps. However, intubation followed by rigid esophagoscopy was also performed in them in order to visualize the status of the esophagus.

Coin was the most common foreign body in the pediatric population and was seen in $82.1 \%$ of cases. In a four year old child, 2 coins of different size were removed on rigid esophagoscopy. Plain X-ray showed a circular radiopaque shadow with two rims of different size plastered together in the lateral view (figure 1).

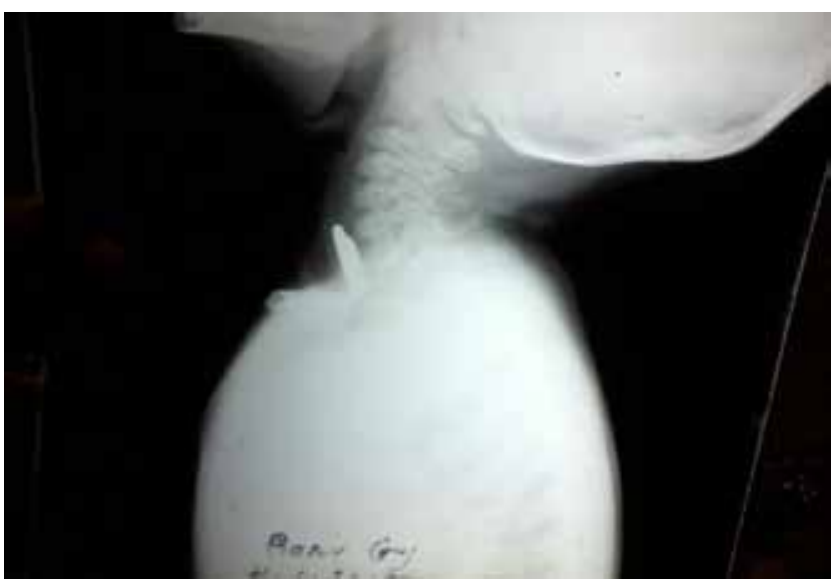

Figure 1.Plain X-ray soft tissue neck lateral view showing two coins in the esophagus. Two rims are visualized plastered to each other.

Bone was seen in $10.7 \%$ cases whereas ear ring and paper clip was seen in $3.5 \%$ cases each.

In the adult population, by contrary, bones were the most common foreign bodies seen in $91.4 \%$ cases. Artificial denture, food particle (orange piece with seeds found in a patient with carcinoma of the esophagus) and variceal band was found to have caused obstruction of the esophagus in $3.1 \%$ of the cases each.

Repeated foreign body ingestion was noted in 3 patients out of which 2 were in pediatric and one in adult. A male patient of 67 years, diagnosed case of carcinoma esophagus and post- radiotherapy with esophageal stricture had it for the second time; a mentally retarded girl of 13 years had the three incidences; and a 3 years old girl with repair of congenital tracheo-esophageal fistula had five repeated incidences of foreign body esophagus.

In the pediatric population, $92.8 \%$ of all the FB got stuck in the upper esophagus while in adults $25.7 \%$ were stuck in upper esophagus and the difference was statistically significant $(\mathrm{P}<0.001)$. Similarly, $7.1 \%$ of the FB was stuck in the mid esophagus in the pediatric population and $65.7 \%$ in adult population $(\mathrm{P}<0.001)$. In the lower esophagus there were $8.5 \%$ of FB stuck in adult population whereas no such incidence was noted in pediatric population $(\mathrm{P}=0.247)$ (Table 1).

Table 1. Comparison of site of impaction of FB in adult and pediatric population (Fischer Exact Test)

\begin{tabular}{lccc}
\hline $\begin{array}{c}\text { Site of Foreign } \\
\text { Body }\end{array}$ & $\begin{array}{c}\text { Children } \\
(\%)\end{array}$ & $\begin{array}{c}\text { Adult } \\
(\%)\end{array}$ & P value \\
\hline Upper esophagus & $26(92.8)$ & $9(25.7)$ & $<0.001$ \\
Mid esophagus & $2(7.1)$ & $23(65.7)$ & $<0.001$ \\
Lower esophagus & $0(0)$ & $3(8.5)$ & 0.247 \\
\hline
\end{tabular}

\section{Discussion:}

Foreign body (FB) impaction in adults and pediatric population differs in various aspects. The main differences noted in this study were regarding type of foreign body, site of impaction and relative difficulties of removal.

In the pediatric population, coins were the most common foreign bodies in our series seen in $82.1 \%$ of cases. In many pediatric series coins were the commonest FB in the esophagus with the range of $76-88 \% .^{1-4,6}$ Contrary in the adult population bones were the most common foreign bodies that got stuck in the esophagus. Most of the studies in the adult population have shown that bones are the most common foreign bodies to be impacted in the esophagus. ${ }^{5-7}$ 
In $91.4 \%$ of our adult populations, foreign bodies in the esophagus were bones. Other foreign bodies were denture, food particle and variceal band.

Neglected foreign bodies are also not uncommon in the pediatric population. Children having the common habit of putting things in their mouth will swallow the foreign body that might get stuck in the esophagus without being noticed by the parents. In our study, a child of 2 years was brought with a neglected foreign body in the esophagus. The child was drooling most of the times and was not showing interest in sucking his mother's milk for one week. The child was first evaluated by the pediatrician and was referred for evaluation of the throat condition. Throat being normal on examination, an x-ray soft tissue neck lateral and AP view was ordered and round foreign body was revealed which turned out to become a coin.

There was also a difference noted in the site of impaction of foreign bodies in adult and pediatric populations. In the pediatric population, $92.8 \%$ of all the FB got stuck in the upper esophagus while in adults $25.7 \%$ were stuck in upper esophagus and the difference was statistically significant $(\mathrm{P}<0.001)$. Similarly, $7.1 \%$ of the FB was stuck in the mid esophagus in the pediatric population and $65.7 \%$ in adult population $(\mathrm{P}<0.001)$. In the lower esophagus there were $8.5 \%$ of FB stuck in adult population whereas no such incidence was noted in pediatric population $(\mathrm{P}=0.247)$. Overall $78 \%$ of all foreign bodies got stuck in the upper esophagus, $17 \%$ in the mid esophagus and 5\% in the lower esophagus. Upper esophagus was the commonest site of foreign body impaction in children and mid esophagus was the commonest site of FB impaction in adults. A review of 400 cases of foreign body esophagus has also described findings similar to our series. ${ }^{5}$ This might be due to the fact that coin becoming more flat and esophagus being narrower, coins might have been impacted at the cricopharyngeal junction.

The next difference was noted in the relative easiness in the technique of removal. Coins being at a higher level were easier to remove. Bones being at a lower level and being sharp were difficult to remove than coins. Bones can cause trauma to esophagus but the coins do not cause trauma. Most of the foreign bodies $(92.8 \%)$ in children, were located in the cricopharyngeal junction (upper esophagus) and thus were relatively easier for removal. Three of such coins located in upper oesophagus were removed by the anesthesiologists using Magill's forceps during intubation. There are reports where coins are regularly removed by Magill forceps under anesthesia as the coin does not cause trauma to the esophagus itself. ${ }^{8}$

Beginners in ENT can learn removing the coins in the esophagus before going to bone removal due to the same fact.
Repeated FB ingestion is common in pre-existing narrow esophageal lumen or mentally impaired persons. Two of our patients had repeated foreign body impaction due to narrow esophageal lumen, one adult and one child. The child had repeated FB impaction due to mental retardation.Parents or caretakers have to be much careful in these situations. They should suspect the foreign bodies when there is difficulty in swallowing and drooling of saliva basically in mentally retarded individuals.

Although many studies have shown male and female populations to have almost equal incidence in FB impaction in the esophagus, our study showed females to have increased incidence in both the population groups. ${ }^{5}$ In our study $62 \%$ of all foreign bodies were impacted in females.

\section{Conclusion:}

There are differences in various aspects of impacted FB esophagus in adult and pediatric populations. Coin being the commonest foreign bodies in children, are relatively easier to remove due to their higher location and less chances of trauma. Contrary in the adults, bones being the commonest foreign body are difficult to remove due to the lower location and chances of trauma.

\section{References:}

1. Schunk JE, Harrison AM, Corneli HM, et al. Fluoroscopic foley catheter removal of esophageal foreign bodies in children: experience with 415 episodes. Pediatrics 1994;94:709-14.

2. Nandi P, Ong GB. Foreign body in the oesophagus: review of 2394 cases. Br J Surg 1978;65:5-9.

3. Little DC, Shah SR, St Peter SD, et al. Esophageal foreign bodies in the pediatric population: our first 500 cases. J Pediatr Surg 2006;41:914-8.

4. Shivakumar AM, Naik AS, Prashanth KB, et al. Foreign body in upper digestive tract. Indian J Pediatr 2004;71:689-93.

5. Athanassiadi K, Gerazounis M, Metaxas E, et al.Management of esophageal foreign bodies: a retrospective review of 400 cases. Eur J Cardiothorac Surg 2002;21:653-6.

6. Kay M, Wyllie R .Pediatric foreign bodies and their management. Curr Gastroenterol Rep 2005;7:212-8.

7. Sittitrai P, Pattarasakulchai T, Tapatiwong H. Esophageal foreign bodies. J Med Assoc Thai 2000;83:1514-8.

8. Janik JE, Janik JS. Magill forceps extraction of upper esophageal coins. J Pediatr Surg 2003;38:227-9. 